\title{
Thermal vacuum chamber repressurization with instrument purging
}

\author{
Michael S. Woronowicz*
}

Stinger Ghaffarian Technologies, Inc., 7701 Greenbelt Rd., Greenbelt, Maryland 20770

\begin{abstract}
At the conclusion of cryogenic vacuum testing of the James Webb Space Telescope Optical Telescope Element Integrated Science Instrument Module (JWST-OTIS) in NASA Johnson Space Center's (JSCs) thermal vacuum (TV) Chamber A, contamination control (CC) engineers are postulating that chamber particulate material stirred up by the repressurization process may be kept from falling into the Integrated Science Instrument Module (ISIM) interior to some degree by activating instrument purge flows over some initial period before opening the chamber valves. This manuscript describes development of a series of models designed to describe this process. The models are strung together in tandem with a fictitious set of conditions to estimate overpressure evolution from which net outflow velocity behavior may be obtained. Creeping flow assumptions are then used to determine the maximum particle size that may be kept suspended above the ISIM aperture, keeping smaller particles from settling within the instrument module.
\end{abstract}

Keywords: contamination control, purging, backfill, repressurization, venting

\section{INTRODUCTION}

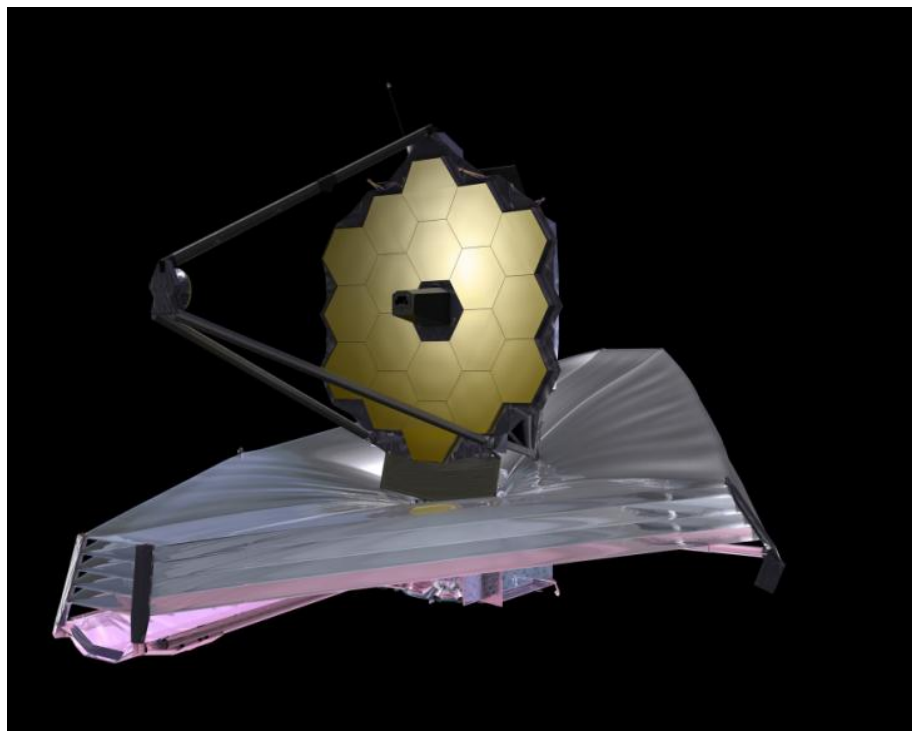

Figure 1. Illustration of the James Webb Space Telescope. ${ }^{1}$

At the end of James Webb Space Telescope (JWST) OTIS (Optical Telescope Element-OTE-Integrated Science Instrument Module-ISIM) Cryovac testing in NASA Johnson Space Center's (JSC's) thermal vacuum (TV) Chamber A, contamination control (CC) engineers are mooting the idea that particulates stirred up by the repressurization process may be kept from reaching the ISIM interior to some degree by first activating instrument purge flows for a set period before opening the chamber pressurizing valves. The ISIM aperture faces upward along the TV chamber's vertical axis.

*michael.woronowicz@nasa.gov; phone 1301 286-5613; fax 1301 286-1704; sgt-inc.com 
This paper describes development of a series of models designed to describe the overall process as the gas transits various domains of rarefaction and other phases. These models are strung together in tandem to estimate overpressure evolution from which net outflow velocity behavior may be obtained. Creeping flow assumptions due to Stokes ${ }^{2}$ are then used to determine the maximum particle size that may be lofted, keeping smaller particles from settling within the ISIM compartment.

\section{MASS CONSERVATION STATEMENT}

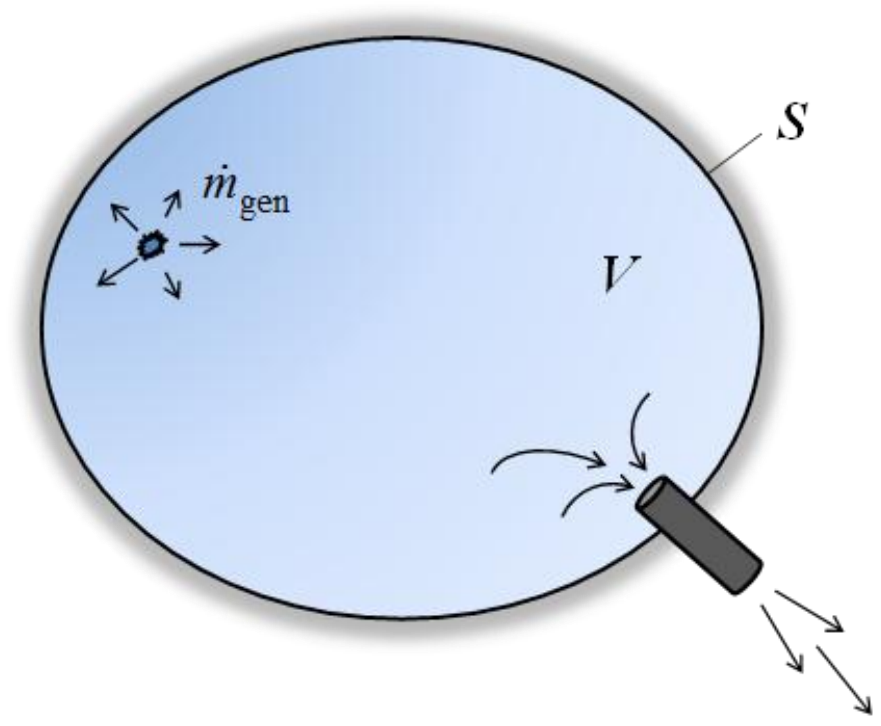

Figure 2. Schematic representation of a simple rigid vessel venting system.

For this application, the rate of mass accumulation for a gaseous species within rigid ISIM volume $V$ is made up of the rate of mass generation $\dot{m}_{\text {in }}$ due to purging minus the net rate at which mass flux exits across that volume's bounding surface $S$. The system is visualized in Fig. 2. Time is represented by $t$, mass density by $\rho$, and local flow velocity by $\boldsymbol{U}$.

$$
\frac{d}{d t} \iiint_{V} \rho d V=\dot{m}_{\mathrm{in}}-\oiint_{S} \rho \boldsymbol{U} \cdot \boldsymbol{d} \boldsymbol{S} .
$$

Assuming thermodynamic conditions within this unit may be lumped, then the volume integral may be replaced by $V$. For an ideal gas under isothermal conditions, Eq. (1) may be simply recast in terms of pressure $p$,

$$
V \frac{d p}{d t}=\dot{m}_{\mathrm{in}} R T-\oiint_{S} p \boldsymbol{U} \cdot \boldsymbol{d} \boldsymbol{S} .
$$

In Eq. (2), species gas constant $R$ for average molar mass $M$ is related to the universal gas constant $R_{\mathrm{u}}$ by $R \equiv R_{\mathrm{u}} / M$. Considering molecular nitrogen as the purge gas, $M=28 \mathrm{~g} / \mathrm{mole}$. The equation now has units of gas load $Q$, often presented in Torr $\cdot \mathrm{L} / \mathrm{s}$ or W. ${ }^{3}$ Since $\dot{m}=\rho G$ where $G$ represents a local volumetric flow rate,

$$
Q=\dot{m} R T=\rho G R T=p G
$$




\subsection{Venting}

Where venting occurs across $k$ orifices in parallel with areas $A_{k}$ against a background pressure $p_{b}$, the surface integral may be replaced by a sum of $K$ conductive terms. ${ }^{3}$

$$
V \frac{d p}{d t}=\dot{m}_{\mathrm{in}} R T-\sum_{k}^{K} F_{k}\left(p-p_{b}\right)
$$

Conductance $F$ has units identical to volumetric flow rate, but is defined using a pressure difference across the vessel boundary. For a single vent out the ISIM aperture, one may dispense with the summation notation.

For states $1 \& 2$ describing conditions within $V$ and the background outside, ${ }^{3}$

$$
F_{1-2} \equiv \frac{Q}{p_{1}-p_{2}}=\frac{p_{1} G}{p_{1}-p_{2}}
$$

Equation 5 will be used to develop relevant venting terms as the repressurization process transits different flow regimes.

\subsection{Venting Equations}

Analysis of this arrangement actually requires describing a number of stages tied together over time in series by various physical constraints. By purging through the instruments, this feature is meant to ensure the pressure will always be higher within the ISIM volume than outside of it even once the vacuum chamber valve is opened (an assumption reviewed in Section 3.4). A positive overpressure will produce a net outward flow across the upward-facing instrument module aperture, preventing particles below a certain size from dropping into ISIM. The average velocity associated with this overpressure may be determined by application of an energy conservation statement. If the net flow velocity is exceedingly low however, one may determine that the maximum particle size lofted against gravity will be so small that the purge application would be considered ineffective.

In this application it is assumed that the instrument purge is initiated one hour before opening TV chamber repressurization valves. The aggregate purge flow rate $G_{I}$ through the instruments is fixed and the ISIM volume is denoted by $V_{I}$. The gas load produced by this purge is assumed to be a constant $Q_{I}$.

Subscript $A$ represents TV Chamber A parameters. Once chamber valves are opened, the overall chamber pressure level $p_{A}$ will increase at a rate of $0.0125 \mathrm{Torr} / \mathrm{s}$. The initial chamber pressure is assumed to lie at an insignificant $10^{-6}$ Torr.

Using the development presented above, the equations used to describe ISIM overpressure in each stage beginning with high vacuum conditions is given by

$$
\begin{aligned}
V_{I} \frac{d p_{I}}{d t} & =Q_{I}-F\left(p_{I}-p_{A}\right) \\
V_{A} \frac{d p_{A}}{d t} & =Q_{A}+F\left(p_{I}-p_{A}\right)
\end{aligned}
$$

For each of the stages identified during the chamber refill process, various physical constraints will produce different expressions for gas loads $Q$ and conductance $F$ linking ISIM venting to Chamber A. Note for Chamber A that there is no venting term because pumping is disengaged, and the venting term describing ISIM pressure feeds into this volume.

In the following development, parameters have been chosen for illustrative purposes only, and do not accurately represent the ensemble associated with the actual ISIM purge/repressurization application. 


\section{PIECEWISE OVERPRESSURE SOLUTIONS}

\subsection{Molecular Flow}

The first stage of this process is assumed to occur under molecular flow conditions (free molecule-FM). FM flow is governed by Knudsen number $K n$, a dimensionless ratio that compares the mean free path length $\lambda$, a characteristic distance between intermolecular collisions, with some characteristic distance $d$ between surfaces. ${ }^{4}$ For continuum conditions in evacuated vessels, an approximate limit is $K n<0.01$. Theoretically, FM conditions dominate for $K n>1.0$. In this application, we will arbitrarily assume FM conditions apply until $K n=0.01$ at the ISIM aperture, where $d_{\text {eff }}=$ $30 \mathrm{~cm}$, ignoring variations that could occur in the transition regime described by $0.01<K n<1$. Based on the hard sphere intermolecular potential with a molecular diameter of $3.75 \AA$ for molecular nitrogen, ${ }^{4,5}$ the crossover to continuum conditions will occur at $\lambda=3.0 \mathrm{~mm}$, which corresponds to a pressure level of 0.017 Torr.

For the first hour, repressurization will occur via instrument purges alone, so $Q_{A}=0$ in (6). Assume the purge rate occurs at $1200 \mathrm{~L} / \mathrm{hr}$ at a supply pressure of two atm, so $Q_{I}=506 . \overline{6}$ Torr L/s. If $V_{A}=10000 \mathrm{~m}^{3}$ while $V_{I}=40 \mathrm{~m}^{3}$ :

$$
V_{A} \frac{d p_{A}}{d t}=Q_{I}-V_{I} \frac{d p_{I}}{d t} \approx Q_{I} ; \quad \dot{p}_{A}=\frac{Q_{I}}{V_{A}}
$$

The chamber repressurization rate under these circumstances is about $\dot{p}_{A}=5.1 \times 10^{-5} \mathrm{Torr} / \mathrm{s}$. Substituting into the equation for ISIM, we find

$$
V_{I} \frac{d p_{I}}{d t}=Q_{I}-F\left(p_{I}-\dot{p}_{A} t\right)
$$

In FM flow, conductance $F=A \sqrt{R T / 2 \pi}$ is not a function of pressure. ${ }^{3}$ The solution for (8) is

$$
p_{I}(t)=p_{I, 0} e^{-\frac{t}{\tau}}+\left(q-\dot{p}_{A}\right) \tau\left(1-e^{-\frac{t}{\tau}}\right)+\dot{p}_{A} t, \quad \text { where } \quad(\tau, q) \equiv\left(\frac{V_{I}}{F}, \frac{Q_{I}}{V_{I}}\right)
$$

Eq. (9) is meant to describe the passage of the environment around the ISIM aperture from true molecular flow all the way to the continuum regime. Even so, the $K n=0.01$ aperture condition is violated a mere $1.5 \mathrm{~s}$ after purge initiation!

\subsection{Continuum--Sonic Constraint}

Once the configuration reaches continuum conditions, ISIM venting is constrained by sonic conditions at the AOS aperture (Mach number $M=1$ ), and is not affected by chamber conditions as long as the pressure level there remains below a certain critical fraction of the ISIM interior pressure created by the purge gases. Assuming isentropic conditions, ${ }^{6}$

$$
\frac{p_{I}}{p_{A}}=\left(1+\frac{\gamma-1}{2} M^{2}\right)^{\frac{\gamma}{\gamma-1}}
$$

For diatomic gases such as molecular nitrogen at room temperature, the ratio of specific heats $\gamma=7 / 5$. For sonic conditions, the maximum chamber pressure that can be supported occurs when $M=1$ and $p_{A}=0.528 p_{I}$. With a constant purge flow rate, we may still assume $p_{A}=\dot{p}_{A} t$. In Eq. (6), the ISIM venting term is replaced by ${ }^{6}$ 


$$
Q_{I, \text { out }}=\dot{m} R T=p_{I} A \sqrt{\gamma R T}\left(\frac{2}{\gamma+1}\right)^{\frac{\gamma+1}{2(\gamma-1)}}=\zeta p_{I} .
$$

Substituting into Eq. (6),

$$
V_{I} \frac{d p_{I}}{d t}=Q_{I}-\zeta p_{I}
$$

The solution becomes

$$
p_{I}(\Delta t)=p_{I, 02} e^{-\frac{\Delta t}{\tau_{s}}}+\frac{Q_{I}}{\zeta}\left(1-e^{-\frac{\Delta t}{\tau_{s}}}\right), \quad \text { where } \tau_{s} \equiv \frac{V_{I}}{\zeta}
$$

The initial pressure for this stage (subscript 02) is given by the $K n=0.01$ value at the end of the previous FM stage, and the $\Delta t$ represents time elapsed from then. These conditions hold until

$$
p_{A, 02}+\dot{p}_{A} \Delta t=0.528 p_{I}, \text { where } \quad p_{I}=f(\Delta t) .
$$

Calculations performed for these example conditions indicate sonic conditions last for about 6.3 minutes after purge initiation, when $p_{I}=0.036$ Torr. Beyond this point, rising chamber back pressure influences ISIM internal pressure as conditions become subsonic.

\subsection{Subsonic, Instrument Purge Only}

Chamber pressure affects ISIM interior pressure through the vent conductance term in Eq. (6) for subsonic conditions: ${ }^{6}$

$$
F=\sqrt{\frac{2 \gamma R T}{\gamma-1}} \frac{p_{I} A}{p_{I}-p_{A}}\left(\frac{p_{A}}{p_{I}}\right)^{\frac{1}{\gamma}} \sqrt{1-\left(\frac{p_{A}}{p_{I}}\right)^{\frac{\gamma-1}{\gamma}}} .
$$

It appears best to solve Eq. (6) numerically. Using superscript $n$ to denote conditions at elapsed time $\Delta t=n \delta t$ :

$$
p_{I}^{n}(n \delta t)=p_{I}^{n-1}+\frac{Q_{I} \delta t}{V_{I}}-\frac{p_{I}^{n-1} A \delta t}{V_{I}} \sqrt{\frac{2 \gamma R T}{\gamma-1}}\left(\frac{p_{A}^{n}}{p_{I}^{n-1}}\right)^{\frac{1}{\gamma}} \sqrt{1-\left(\frac{p_{A}^{n}}{p_{I}^{n-1}}\right)^{\frac{\gamma-1}{\gamma}}},
$$

where

$$
p_{A}^{n}(n \delta t)=p_{A, 03}+\dot{p}_{A} n \delta t .
$$

Initial conditions in this case (subscript 03) are taken from the previous stage at the instant sonic conditions were breached. This calculation was performed out to the one hour mark. Using a constant timestep, calculations had to iterate in order to produce an acceptably small timestep ( $\delta t=0.2 \mathrm{~s}$ ) so that the last term in Eq. (11) would not begin causing the entire solution to become unstable, oscillate, and quickly produce physically unrealistic values. By the end of this calculation, chamber pressure has risen to 0.1834 Torr, which is only $3.2 \mathrm{e}-5$ psig below that for ISIM. 


\subsection{Subsonic, Instrument Purge \& Chamber Valves}

As just mentioned, by the end of that first hour before chamber valves are opened and a new source has been added to the system, calculations indicate that overpressure $\Delta p \equiv p_{I}-p_{A}$ has already become a small fraction of either pressure alone. This fraction will decrease even further as direct chamber repressurization comes into play, and requires smaller timesteps to prevent the instability discussed above. However it turns out that a tremendous simplification can come into play when $\Delta p / p<0.1$.

In small disturbance theory for $\varepsilon=\Delta p / p<<1$, one may use approximations such as $(1+\varepsilon)^{a} \approx 1+a \varepsilon$ and neglect $\mathrm{O}\left(\varepsilon^{2}\right)$ and higher to greatly simplify Eq. (15): ${ }^{7}$

$$
F_{\mathrm{sm}} \equiv F\left(\Delta p<<p_{A}\right) \approx A \sqrt{2 R T \frac{p_{A}}{\Delta p}}\left(1-\frac{1}{\gamma} \frac{\Delta p}{p_{A}}\right) \approx A \sqrt{2 R T \frac{p_{A}}{\Delta p}} .
$$

Notice in Eq. (18) that conductance $F_{\mathrm{sm}}$ is a function of both $p_{I}$ and $p_{A}$, but in this incompressible small disturbance limit it is independent of $\gamma$, hence it no longer depends on the atomic structure of the gas species under consideration.

Also, the chamber fill rate has increased considerably from about $5.1 \times 10^{-5} \mathrm{Torr} / \mathrm{s}$ to $\dot{p}_{A, 4}=1 \mathrm{Torr} / \mathrm{min}=1.25 \times 10^{-2}$ Torr/s, overwhelming the effect of the purge. For small disturbances, one may assume $\dot{p} \equiv \dot{p}_{I, 4}=\dot{p}_{A, 4}$, which is constant. This turns the non-linear ordinary differential equation for $p_{I}$ in Eq. (6) into an algebraic statement that may be solved analytically.

$$
\begin{gathered}
V_{I} \dot{p}=Q_{I}-A \sqrt{2 R T} \sqrt{p_{A}\left(p_{I}-p_{A}\right)} \\
p_{A}(\Delta t)=p_{A, 04}+\dot{p} \Delta t
\end{gathered}
$$

The solution for $p_{I}$ becomes

$$
p_{I}=p_{A}+\frac{1}{2 R T p_{A}}\left(\frac{Q_{I}-V_{I} \dot{p}}{A}\right)^{2}
$$

Equations $19 \& 20$ were used to describe the chamber environment up to the point where $p_{A}=760$ Torr and repressurization was completed. Notice the gas load terms in the numerator of Eq. (20), which represent the strengths of sources within ISIM versus that outside of it. This condition must be evaluated to determine if it matches the proper physics. It turns out that $506 . \overline{6}-500$ Torr L/s is positive (barely), which means the net flow is out of ISIM. Although Eq. (20) would still be positive if the external source were higher than the inner one, Eq. (18) would need to be recast in terms of $p_{I}$ and $\Delta p=p_{A}-p_{I}$ to reflect the fact that gas would be flowing into ISIM.

The results described in these sections spanning four flow regimes are depicted together in Fig. 3 below, where net overpressure of ISIM above the chamber background is depicted versus time. Early on the overpressure increases with time as the ISIM aggregate instrument purge fills that volume with purge gas to higher pressure levels ahead of its influence on the chamber, although the maximum overpressure achieved is never quite a sensational level $\left(6.8 \times 10^{-4} \mathrm{psi}\right.$ at $15.5 \mathrm{~s}$ after purge initiation). Then the chamber pressure reaches a level where a sonic orifice conditions can no longer be maintained, and soon afterwards the pressure differential settles into an inverse time dependence in subsonic flow. Once the chamber repressurization valves are opened, the ISIM overpressure amplitude falls below the minimum level detectable by ear (about $\left.3 \times 10^{-9} \mathrm{psig}\right){ }^{10}$ 


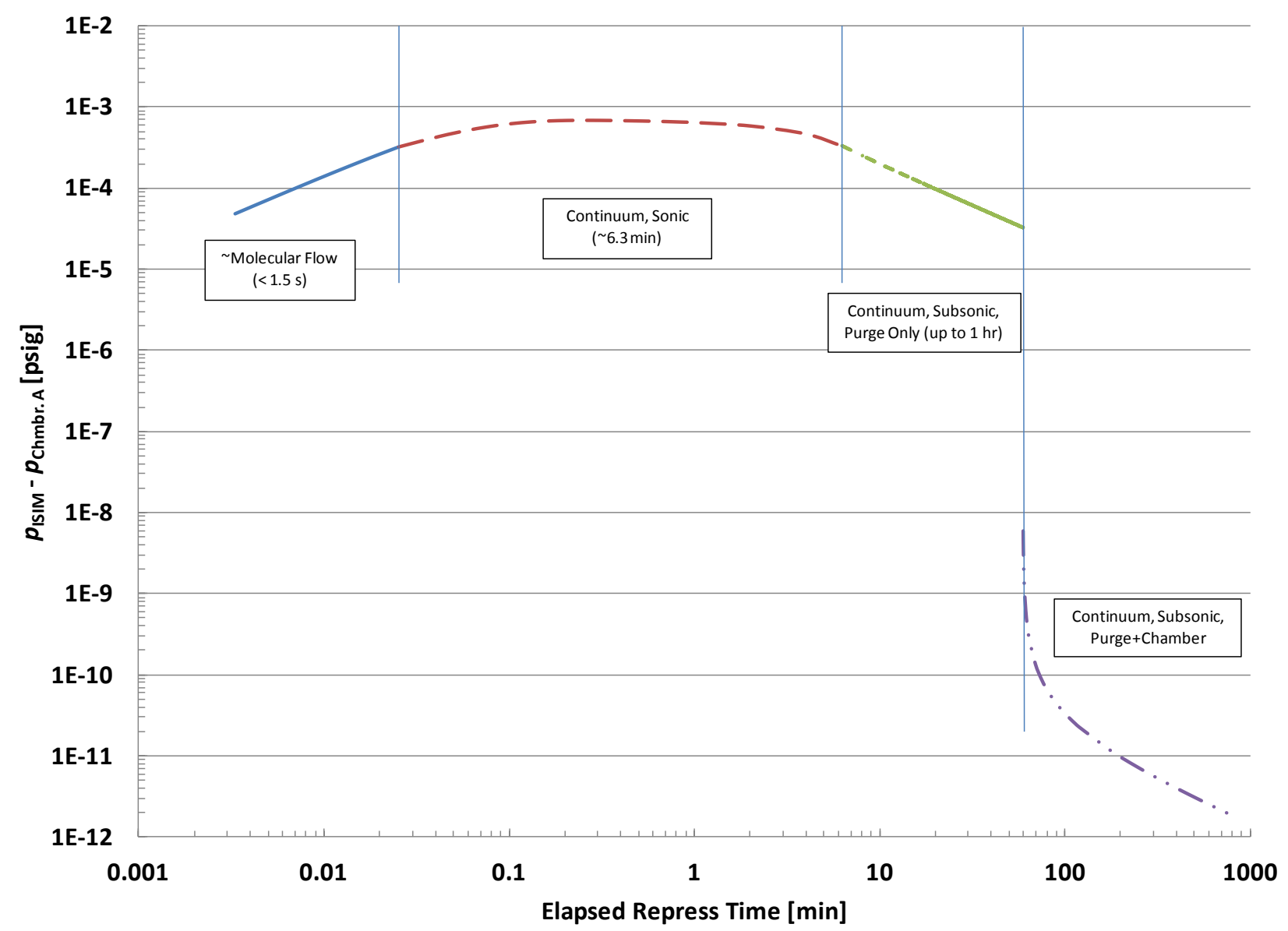

Figure 3. Notional repressurization sequence overpressure spanning four flow regimes.

\section{CREEPING FLOW ANALYSIS}

\subsection{Creeping Flow Assumption}

Since we are investigating particulate matter having length scales on the order of microns immersed in low speed flow, we expect the Reynolds number based on particle diameter to lie at or below $R e=1$, for which creeping flow effects should apply. ${ }^{2}$ Using room temperature values for molecular nitrogen (dynamic viscosity $\mu$ of $1.78 \times 10^{-5} \mathrm{~kg} / \mathrm{m} / \mathrm{s}, \rho=$ $1.14 \mathrm{~kg} / \mathrm{m}^{3}$ at $\left.1 \mathrm{~atm}\right)$,

$$
R e \equiv \frac{\rho U D}{\mu} \leq 1 \rightarrow(U D)_{\text {crit }} \leq\left\{\begin{array}{l}
1.56 \times 10^{-5} \mathrm{~m}^{2} / \mathrm{s} \text { at } 1 \mathrm{~atm} \\
0.065 \mathrm{~m}^{2} / \mathrm{s} \text { at } 0.1834 \text { Torr (see Sect. 3.3) }
\end{array}\right.
$$

Equation (23) reveals the condition for creeping flow, which depends on $p_{A}$. If the aperture velocity estimate was 10 $\mathrm{cm} / \mathrm{s}$, the range of validity for creeping flow would include particles below $D=160$ microns in diameter at one atmosphere, but $65 \mathrm{~cm}$ at the initiation of chamber valve opening! Hence, the creeping flow assumption appears to cover the ranges of anticipated flow velocities and particle sizes for this application. 


\subsection{Net Velocity Calculation}

Once chamber valves are opened, the flow created by this influence is known to stir up particulate matter. Some of these particles could potentially settle inside ISIM after falling in through the vertical aperture. Since the reasoning behind first applying an aggregate instrument purge is meant to reduce the amount of matter entering that volume later, it was decided to convert the overpressure at the aperture to a net velocity by applying an energy conservation statement where ISIM acts like a reservoir (velocity $U_{I} \approx 0$ within the volume for cross-sections much larger than the aperture) and potential energy differences in height working against gravity may also be neglected. It is further assumed this statement applies at each instant in time quasistatically for this incompressible fluid. Across the aperture (subscript ap), as far as these assumptions apply, the statement simplifies to the Bernoulli equation: ${ }^{8}$

$$
\Delta p \equiv p_{I}-p_{A}=\frac{1}{2} \rho U_{a p}^{2}-\frac{1}{2} \rho U_{I}^{2} \approx \frac{1}{2} \rho U_{a p}^{2} .
$$

As this statement is assumed to apply at each instant in time, gas density $\rho$ may be calculated using the ideal gas law. Based on the small disturbance development, the aperture velocity estimate becomes

$$
U_{a p}=\sqrt{2 R T \varepsilon} .
$$

\subsection{Critical Particle Size}

A force balance for lofting a particle with mass $m$ vertically along the $y$ direction with viscous drag in creeping flow against gravity (acceleration $-g$ ) produces

$$
m \ddot{y}=F_{s}-m g \text {. }
$$

For spherical particles, the viscous drag force is given by the Stokes formula. ${ }^{2}$ This formula remains approximately valid for a variety of shapes. ${ }^{2}$

$$
F_{s}=6 \pi \mu R\left(U_{a p}-\dot{y}\right) \text {. }
$$

For critical conditions, a particle having an equivalent radius of $R_{c r i t}$ is suspended without vertical motion when

$$
U_{a p} \equiv \frac{m g}{6 \pi \mu R_{c r i t}}=g \tau
$$

In the general solution to Eq. (25), $\tau$ is identified as an inertial time constant associated with accelerating the particle. For an aluminum particle having a diameter of 100 microns, $\tau=84$ milliseconds. Thus, it appears particle motion should react rapidly to changes in flow conditions and the quasistatic assumption is justified.

Equation 26 may be written in terms of $R_{\text {crit }}$. For a particle having average mass density $\rho_{s}$, the critical radius becomes

$$
R_{c r i t}=\sqrt{\frac{9 \mu U_{a p}}{2 \rho_{s} g}} .
$$

Values of $R_{c r i t}$ and net velocity $U_{a p}$ were plotted versus time from the point where chamber valves were opened in Fig. 4 below. A surprising result based on the use of Eq. (25) is that the particle drag force is not a function of gas density, unlike the case at higher Reynolds numbers. One consequence of this feature is that larger particles may be 
lofted at low density levels because higher overpressures translate to larger velocities $U_{a p}$ ! Another consequence is that the worst case condition occurs at the end of this phase as the chamber just reaches one atmosphere.

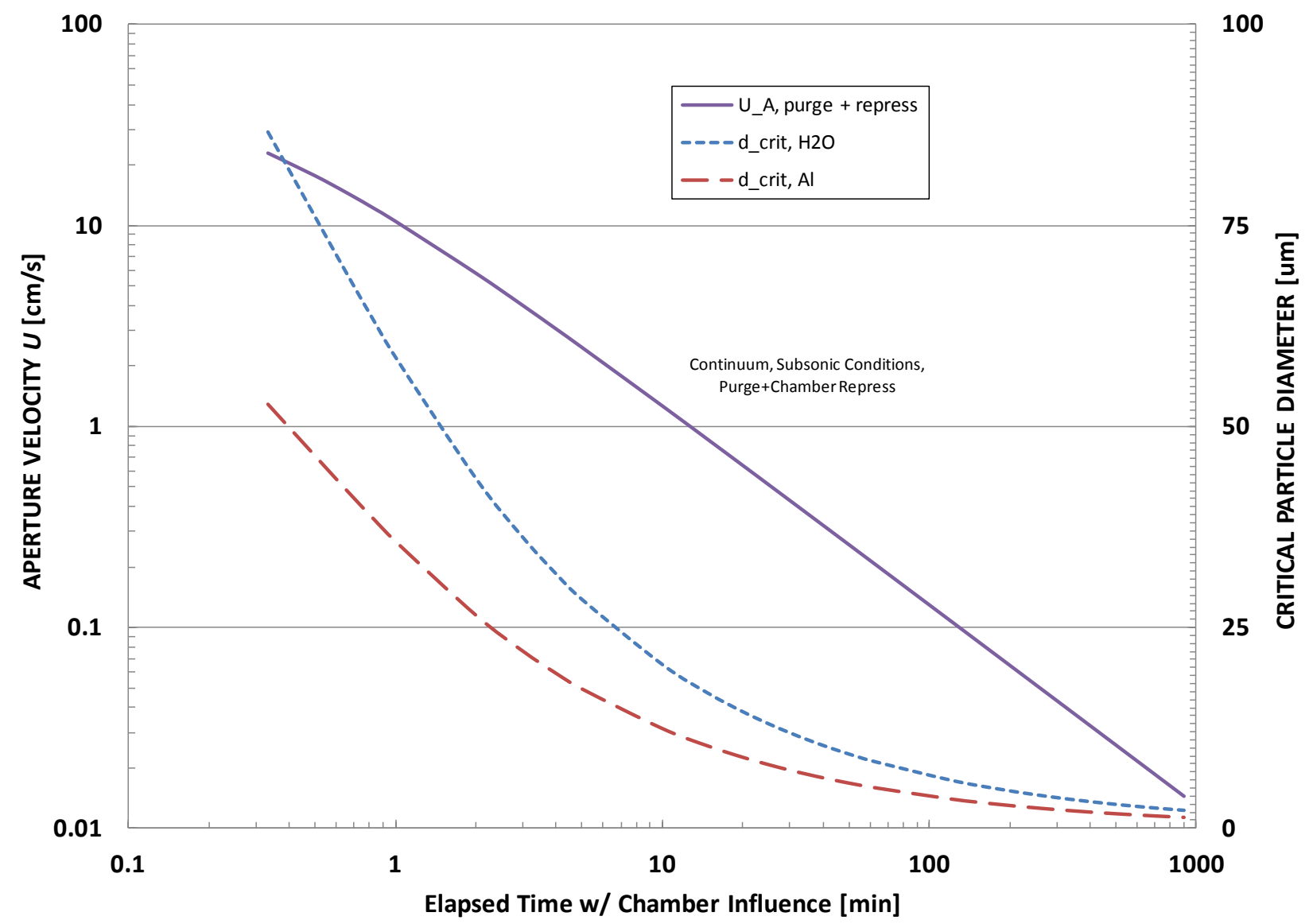

Figure 4. Maximum suspended particle sizes above ISIM aperture during TV repressurization with chamber valve activated.

For particle mass density values similar to water and aluminum $\left(\rho_{s}=1.0 \& 2.7 \mathrm{~g} / \mathrm{cm}^{3}\right){ }^{9}$ predictions indicate the largest particle sizes that may be suspended above the ISIM aperture against the chamber valve influence during repressurization are 2.0 microns and 1.2 microns, respectively. Since particle fallout distributions are heavily skewed towards high concentrations of small elements, these results indicate that it is possible to reject a large fraction of a fallout ensemble by number; however, these deflected particles constitute a relatively small fraction of potential area concealable by such distributions.

\section{CONCLUDING REMARKS}

An analysis was performed to estimate the ability of an instrument purge to keep particulate matter from entering the JWST Integrated Science Instrument Module during chamber repressurization using the facility system. A series of models were developed to describe net overpressure across the instrument module's aperture from molecular flow to continuum conditions, with the process also spanning sonic, subsonic compressible, and finally incompressible environments. This overpressure was translated to determine aperture velocity for evaluating viscous drag on various particle sizes using creeping flow conditions. 
Although the complex set of example conditions used to convey the overall application did not result in a robust ability to deny particulate matter entrance to the instrument module by the end of the repressurization process, this situation may be remedied by increasing the aggregate purge rate (if possible) or by slowing down the chamber repressurization rate.

\section{ACKNOWLEDGEMENTS}

The author gratefully acknowledges support from NASA Contract NNG15CR64C, particularly the encouragement of Ms Eve Wooldridge (NASA GSFC Code 546), and SGT, Inc.

\section{REFERENCES}

[1] Anon., "James Webb Space Telescope," Wikipedia, 31 August 2009, <http://www.jwst.nasa.gov/images_artist 13532.html>, (21 June 2016).

[2] White, F., [Viscous Fluid Flow], McGraw Hill Book Co., New York, 204-210 (1974).

[3] Dushman, S., [Scientific Foundations of Vacuum Technique], John Wiley \& Sons, New York, 81-91 (1962).

[4] Vincenti, W., and Kruger, C., [Introduction to Physical Gas Dynamics], Robert E. Krieger Pub. Co., Malabar, FL, 14 (1982).

[5] Kennard, E., [Kinetic Theory of Gases], McGraw-Hill, New York, 149 (1938).

[6] Oates, G., [Aerothermodynamics of Gas Turbine and Rocket Propulsion, Revised and Enlarged], AIAA Education Series, J. Przemieniecki, ed., American Institute of Aeronautics and Astronautics, Inc., Washington, DC, 50-53 (1988).

[7] Woronowicz, M., “On small disturbance ascent vent behavior," Proc. SPIE 8492, 8492 0E (2012).

[8] Clancy, L., [Aerodynamics], John Wiley \& Sons, New York, 23 (1975).

[9] Kreith, F., and Black, W., [Basic Heat Transfer], Harper \& Row Publishers, New York, 509-527 (1980).

[10] Anon., "Hearing Range," Wikipedia, 26 June 2016, <https://en.wikipedia.org/wiki/Hearing_range>, (5 July 2016). 